\title{
Principal Oscillation Pattern analysis of the tropical 30- to 60-day oscillation
}

\section{Part II: The prediction of equatorial velocity potential and its skill}

\author{
Hans von Storch ${ }^{1}$ and David $\mathbf{P}$ Baumhefner ${ }^{2}$ \\ ${ }^{1}$ Max-Planck-Institut für Meteorologie, Bundestrasse 55, W-2000 Hamburg, Federal Republic of Germany \\ ${ }^{2}$ National Center for Atmospheric Research*, Boulder, CO 80307-3000, USA
}

Received July 16, 1990/Accepted December 10, 1990

\begin{abstract}
In Part I (Storch and Xu 1990) the principal oscillation pattern (POP) analysis of $200 \mathrm{mb}$ equatorial velocity potential leads to the definition of a bivariate (POP-) index of the tropical 30- to 60-day oscillation. Using the POP prediction scheme this index is predictable for a few days in advance. In Part II, the prediction of the equatorial velocity potential field, made by the POP method and made by two GCMs, is investigated. The POP index forecast can incorporate skillful forecasts of the equatorial velocity potential $(\chi)$ field. Its ensemble correlation skill score passes the 0.50 level at 7 days, whereas persistence passes after 3 days. If there is a strong 30- to 60-day oscillation signal in the initial state, useful forecasts of more than 20 days are sometimes possible; if the initial signal is weak, the POP forecast fails. Also, the forecast skill of two GCMs is considered. The NCAR T31 GCM appears to be quite skillful in predicting the equatorial $\chi$-field, and in particular the 30- to 60-day oscillation. Its skill, however, is less than that of the POP scheme. The CNRM T42 GCM seems not to be able to predict the regular development associated with the tropical 30- to 60-day oscillation. The power of the POP index in explaining the equatorial $\chi$-field is a measure of the strength and dominance of the 30- to 60-day oscillation. This measure at day 0 is an a priori indicator of the NCAR T31 GCM's skill in predicting the equatorial velocity potential field.
\end{abstract}

\section{Introduction: the POP index of the 30- to 60-day} oscillation

In Part I (Storch and Xu 1990; hereafter abbreviated as SX) the POP analysis was used to objectively identify the 30- to 60-day oscillation (Madden and Julian 1972)

* The National Center for Atmospheric Research is sponsored by the National Science Foundation

Offprint requests to: $\mathrm{H}$ von Storch in the equatorial velocity potential at 200 mbar. In appeared that the 30 - to 60-day oscillation may conveniently be described by two POP-patterns, $\mathfrak{p}_{1}$ and $\mathfrak{p}_{2}$ (Fig. 1), and their time-dependent POP-coefficients $z_{1}$ and $z_{2}$ which may be regarded as an index of the dynamic state of the oscillation. In the following, we refer to the paired POP coefficient $\left(z_{1}, z_{2}\right)$ simply as the index of the tropical 30- to 60-day oscillation. The index satisfies a linear dynamic equation which describes a dampened oscillatory infinite sequence of the patterns,

$\cdots \rightarrow-\mathfrak{p}_{1} \rightarrow \mathfrak{p}_{2} \rightarrow \mathfrak{p}_{1} \rightarrow-\mathfrak{p}_{2} \rightarrow-\mathfrak{p}_{1} \rightarrow \cdots$

(see also Appendix). This oscillatory behavior is nicely reflected in the complex auto-correlation function $\varrho(\Delta)=\gamma(\Delta) / \gamma(0)$, with $\gamma(\Delta)=\varepsilon\left(\left(z_{1}+i z_{2}(t) \cdot\left(z_{1}-i z_{2}\right)(t+\Delta)\right)\right.$, of the index (Fig. 2) and in the scatter diagram of the bivariate index $\left(z_{1}, z_{2}\right)$ (Fig. 3). $i$ denotes the imaginary unit and $\varepsilon$ the expectation operator. The fact that $\varrho$ (10 days) is almost purely imaginary is interpreted that, on average, the index is rotated clockwise by $90^{\circ}$ after 10 days in the two-dimensional $z_{1} / z_{2}$-plane relative to its initial position (Fig. 2). Similarly the rotation

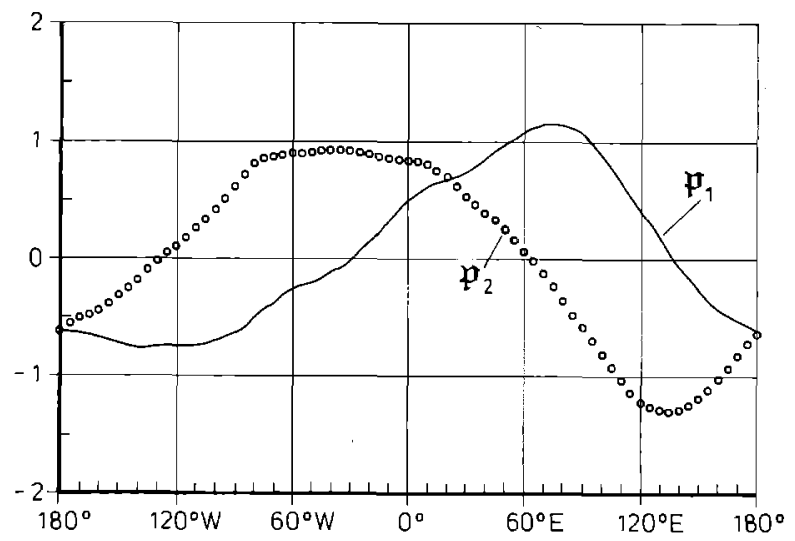

Fig. 1. POP-patterns $p_{1}$ and $p_{2}$ of the tropical 30- to 60-day oscillation as derived by Storch and Xu (1990, abbreviated by SX). The coefficients of these patterns satisfy a linear dynamical equation which describes a dampened oscillatory infinite sequence of the patterns, $\cdots \rightarrow-\mathfrak{p}_{1} \rightarrow \mathfrak{p}_{2} \rightarrow \mathfrak{p}_{1} \rightarrow-\mathfrak{p}_{2} \rightarrow-\mathfrak{p}_{1} \rightarrow \cdots$ 


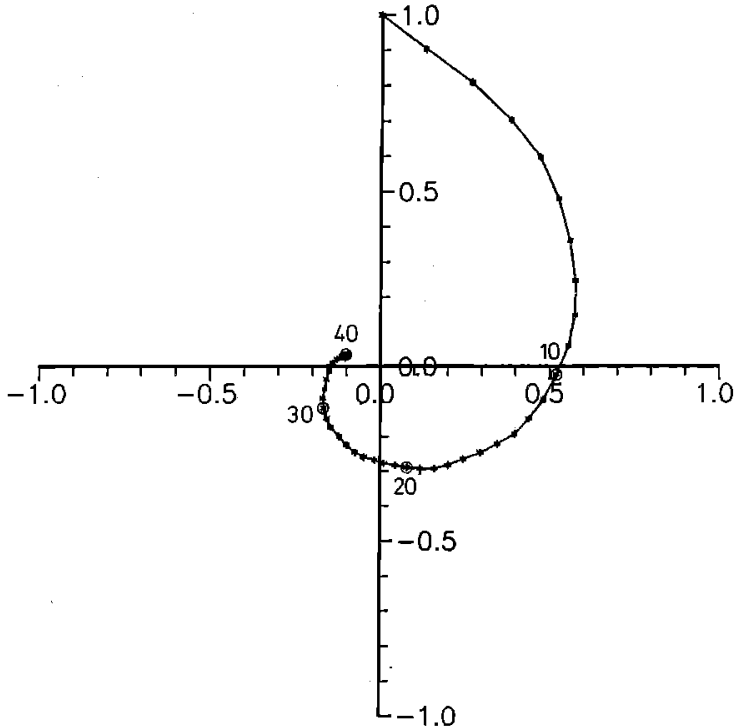

Fig. 2. The complex auto-correlation function $\varrho(\Delta)=\gamma(\Delta) / \gamma(0)$ of the $\left(z_{1}, z_{2}\right)$-index of the tropical 30- to 60-day oscillation. $\gamma(\Delta)$ is the complex auto-covariance function,

$\varepsilon\left(\left(z_{1}+i z_{2}\right)(t) \cdot\left(z_{1}-i z_{2}\right)(t+\Delta)\right)$, with $i$ denoting the imaginary unit and $\varepsilon$ the expectation operator. Vertical axis: real part of $\varrho$, horizontal axis: imaginary part of $\varrho$. The lags $10,20,30$ and 40 days are marked by small circles. The fact that $o(10$ days $)$ is almost purely imaginary indicates that, on average, the index after 10 days is rotated clockwise by $90^{\circ}$ in the two-dimensional $z_{1} / z_{2}$ plane relative to its initial position. Similarly the rotation is $180^{\circ}$ after 22 days and $270^{\circ}$ after 34 days

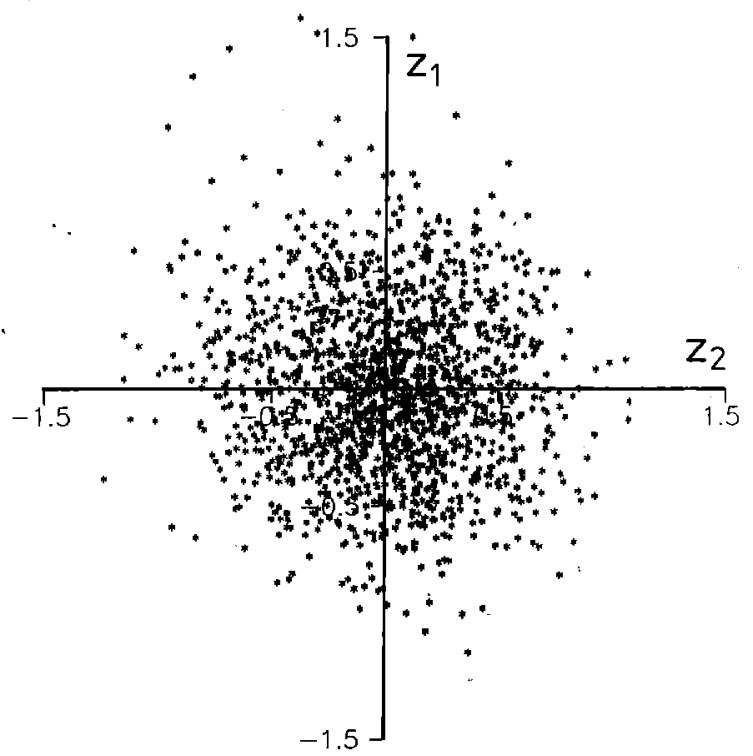

Fig. 3. Scatter diagram of the $\left(z_{1}, z_{2}\right)$-index of the tropical 30- to 60-day oscillation, derived from all available data from January 1985 through April 1989. The distribution of the index is approximately binormal and shows no preference for certain sectors. Units: $10^{6} \mathrm{~m}^{2} \mathrm{~s}^{-1}$ is $180^{\circ}$ after 22 days and $270^{\circ}$ after 34 days. The distribution of the index is approximately binormal and has no preference for certain sectors (Fig. 3).

In SX it was also shown that the index of the 30- to 60-day oscillation may be predicted by the POP forecast scheme (Xu and Storch 1990) for several days. If this is done, the POP forecast scheme is superior to the persistence forecast. The POP index forecast passes the 50\% correlation skill score level after about 10 days.

In the present paper, the usefulness and the skill of the POP forecast scheme is further examined. It is, in particular, extended to allow for predictions of fields, such as the equatorial velocity potential $(\chi)$ and the local outgoing long wave radiation (OLR), that are dominated by the tropical 30- to 60-day oscillation and, thus, are well described by the index (see second section). One might expect useful forecasts of the fields only in those (frequent) situations when the 30- to 60-day oscillation is active.

In the third section, the POP forecasts of the index and of the equatorial velocity potential field are compared with two sets of experimental forecasts which have been prepared by two general circulation models: the T31 NCAR CCM (Baumhefner 1989) and the T42 CNRM model (Coiffier et al. 1987; Geleyn et al. 1988). The question of whether the POP analysis yields a priori clues on the GCMs skill in predicting a certain $\chi$ field is briefly addressed in the fourth section, which discusses the overall results obtained.

The concept of the POP analysis and of the POP forecast are briefly summarized in the Appendix.

In the paper, a possibly confusing number of forecast schemes and of predictants are used. If the predictant is the POP index $\left(z_{1}, z_{2}\right)$ of the tropical 30- to 60day oscillation, the expression POP index ... forecast is used. This forecast is either a persistence forecast or a $P O P$ forecast. If the predictant is the equatorial velocity potential $(\chi)$ at 200 mbar, or outgoing long wave radiation, we refer to it as $\chi$-field or $O L R$... forecast, with the specification persistence, POP, or GCM (NCAR T31 or CNRM T42). Thus, a POP index POP forecast is a forecast of the POP index using the POP forecast sche$\mathrm{me}$, and a $\chi$-field persistence forecast is a prediction of the equatorial velocity potential using persistence.

\section{The POP forecast scheme}

The POP coefficients, $z_{1}$ and $z_{2}$, form a bivariate index, $\mathbf{z}=\left(z_{1}, z_{2}\right)$, of the state of the 30 - to 60 day oscillation (SX). Using the POP forecast scheme (see Appendix; SX) z may be skillfully predicted for a few days in advance. This predictability of the index incorporates a potential to predict the state of (vector) variables which are directly influenced by the 30 - to 60 -day oscillation. If this variable is denoted by $x$ a prediction of its future states requires the knowledge of two states $\mathfrak{r}_{1}$ and $\mathfrak{r}_{2}$ which typically occur if $\mathrm{z}=(1,0)$ and $\mathrm{z}=(0,1)$. If $t$ is the initial date and $\tau$ the forecast lag, and $\hat{\mathbf{z}}(t+\tau)=$ $\left(\hat{z}_{1}(t+\tau), \hat{z}_{2}(t+\tau)\right)$ the POP predicted index, then the POP-forecast $\hat{\mathfrak{f}}(t+\tau)$ is: 
von Storch and Baumhefner: POP analysis of oscillation: Part II -

$\hat{\mathfrak{r}}(t+\tau)=\hat{z}_{1}(t+\tau) \cdot \mathfrak{r}_{1}+\hat{z}_{2}(t+\tau) \cdot \mathfrak{r}_{2}$

Since the POPs were derived from the equatorial velocity potential fields (SX), the POPs $\mathfrak{p}_{1}$ and $\mathfrak{p}_{2}$ (Fig. 1) themselves serve as $\mathfrak{x}_{1}$ and $\mathfrak{x}_{2}$ to predict the equatorial velocity potential $\chi$ (next section). For OLR the "associated correlation patterns" of OLR (see SX) are used. A forecast of the $\chi$-field using Eq. 1 is named a $\chi$-field POP forecast whereas $\hat{z}(t+\tau)$ is a POP index POP forecast.

A measure of the skill of an individual vector forecast is given by the anomaly correlation skill core $\mathscr{\&}$ :

$$
\mathscr{A}(t, \tau)=\frac{\hat{\mathfrak{f}}(t+\tau)^{T} \cdot \mathfrak{r}(t+\tau)}{\sqrt{\|\hat{\mathfrak{f}}(t+\tau)\|\|\mathfrak{d}(t+\tau)\|}}
$$

The symbol ${ }^{T}$ indicates transposition so that the nominator in Eq. 2 is the dot product of the forecast and the verifying analysis at day $t+\tau$. The symbol $\|\mathfrak{r}\|$ refers to the norm of the vector $\mathfrak{x}:\|\mathfrak{r}\|^{2}=\mathfrak{r}^{T} \mathfrak{r}$. The mean anomaly correlation coefficient $\overrightarrow{\mathscr{A}}(\tau)$ is derived by simply averaging the anomaly correlation coefficients from an ensemble of individual forecasts.

Another measure of the overall forecast skill is the correlation skill score $\mathscr{P}$ :

$\mathscr{P}(\tau)=\frac{\left\langle\hat{\mathfrak{r}}^{T}(t+\tau) \cdot \mathfrak{r}(t+\tau)\right\rangle}{\sqrt{\langle\|\hat{\mathfrak{r}}(t+\tau)\|\rangle\langle\|\mathfrak{r}(t)\|\rangle}}$

In contrast to Eq. 2, 3 is defined with ensemble parameters: the brackets, $\langle$.$\rangle , indicate ensemble averages.$

A skill score of $\mathscr{S}=1$ or $\mathscr{A}=1$ indicates a perfect forecast. To establish the lower limits of usefulness of $\mathscr{P}$ and $\mathscr{A}$, we determine the skill of a forecast whose error variance, $\left\langle\|\hat{\mathfrak{x}}-\mathfrak{x}\|^{2}\right\rangle$ or $\|\hat{\mathfrak{x}}-\mathfrak{x}\|^{2}$, is identical to the error variance obtained for the climatological forecast $(\hat{\mathfrak{f}}=0)$ (Murphy and Epstein 1989; Tribbia and Baumhefner 1988):

$$
\begin{aligned}
& \left\langle\|\hat{\mathfrak{x}}-\mathfrak{r}\|^{2}\right\rangle=\left\langle\|\mathfrak{r}\|^{2}\right\rangle \quad \text { or }\|\hat{\mathfrak{r}}-\mathfrak{r}\|^{2}=\|\mathfrak{r}\|^{2} \\
& \text { Then } 2\left\langle\hat{\mathfrak{x}}^{T} \mathfrak{x}\right\rangle=\left\langle\|\hat{\mathfrak{x}}\|^{2}\right\rangle \quad \text { or } 2 \hat{\mathfrak{x}}^{T} \mathfrak{x}=\|\hat{\mathfrak{x}}\|^{2} \\
& \text { and } \mathscr{S}_{\min }=\frac{1}{2} \sqrt{\left\langle\|\hat{\mathfrak{x}}\|^{2}\right\rangle /\left\langle\|\mathfrak{r}\|^{2}\right\rangle} \text { or } \mathscr{S}_{\min }=\frac{1}{2} \sqrt{\|\mathfrak{r}\|^{2} /\|\mathfrak{x}\|^{2}} \text {. }
\end{aligned}
$$

Thus, if the variance of the ensemble of all forecasts, $\left\langle\|\hat{\mathfrak{x}}\|^{2}\right\rangle$, or the spatial variance of an individual forecast, $\|\hat{\mathfrak{x}}\|^{2}$, is identical to the variance of the respective verifying quantity, $\left\langle\|\mathfrak{r}\|^{2}\right\rangle$ or $\|\mathfrak{r}\|^{2}$, the lower limits, $\mathscr{P}_{\text {min }}$ and $\mathscr{A}_{\text {min }}$, for useful forecasts are 0.5 . This condition is fulfilled by the POP forecasts used in this study.

To check the physical skill introduced by the POP model, the skill scores are compared with a trivial forecast scheme, the persistence $\hat{\mathfrak{f}}(t+\tau)=\mathfrak{t}(t)$.

\section{POP forecasts of the equatorial velocity potential field}

The 30- to 60-day oscillation describes the bulk of the daily variance of equatorial velocity potential $\chi$ in $200 \mathrm{mb}$ (SX). Since the index of the oscillation is predictable with the POP scheme, it is reasonable to expect useful $\chi$-field POP forecasts, as long as the 30 - to 60 day oscillation is active.

In this section we consider first, a set of randomly chosen cases, and then the overall skill as given by the correlation skill score $\mathscr{P}$. The annual cycle has been removed from all data and only anomalies are considered.

Case studies

In SX, the POP index and its POP prediction were examined in some detail for 10 randomly chosen cases, denoted by $\mathrm{A}$ to $\mathrm{J}$, from 1985 . These cases are re-examined here with respect to the prediction of the equato-

Table 1. Anomaly correlation coefficients $\mathscr{Z}(t, \tau)$ of $\chi$-field POP forecasts in ten randomly chosen cases from 1985 . These ten cases have been examined already in SX. If no verification data is available, a "**" is given. If there is an initial interval with $\mathscr{A} \geq 50 \%$ the numbers are given in italics, indicating a skillful forecast

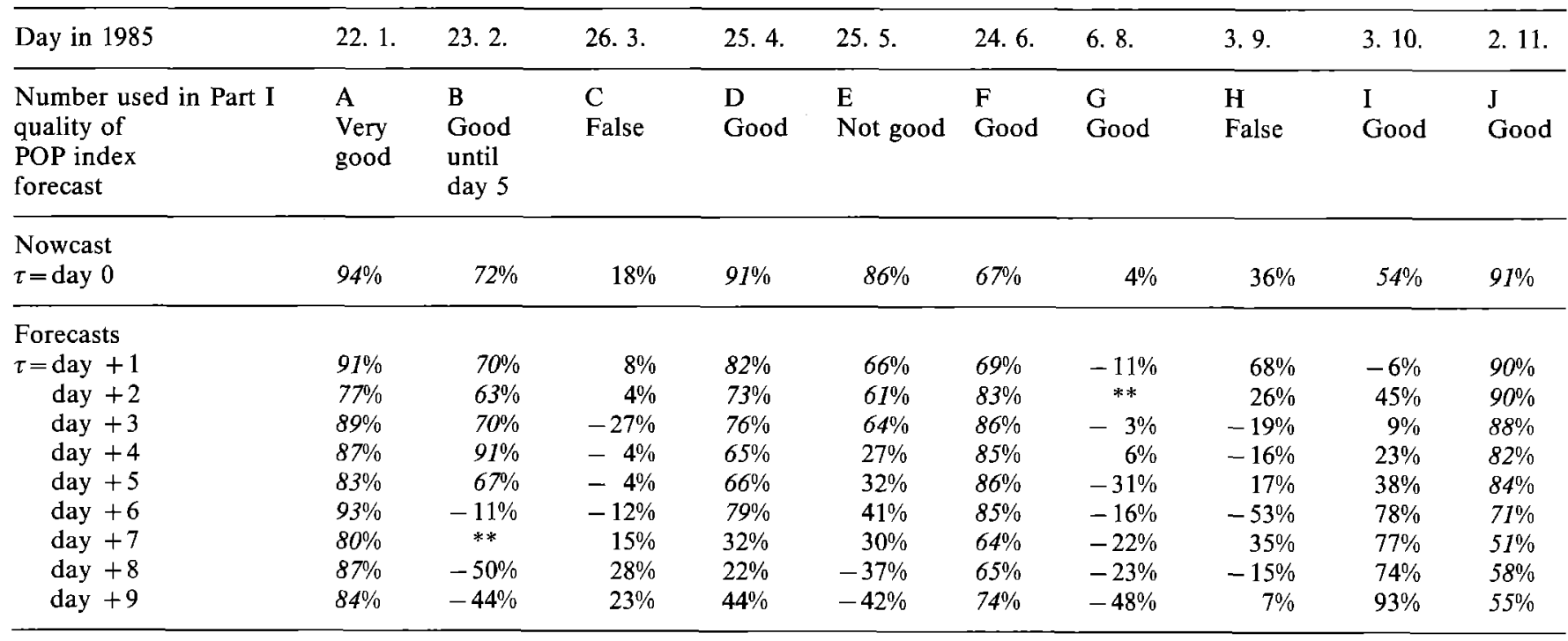



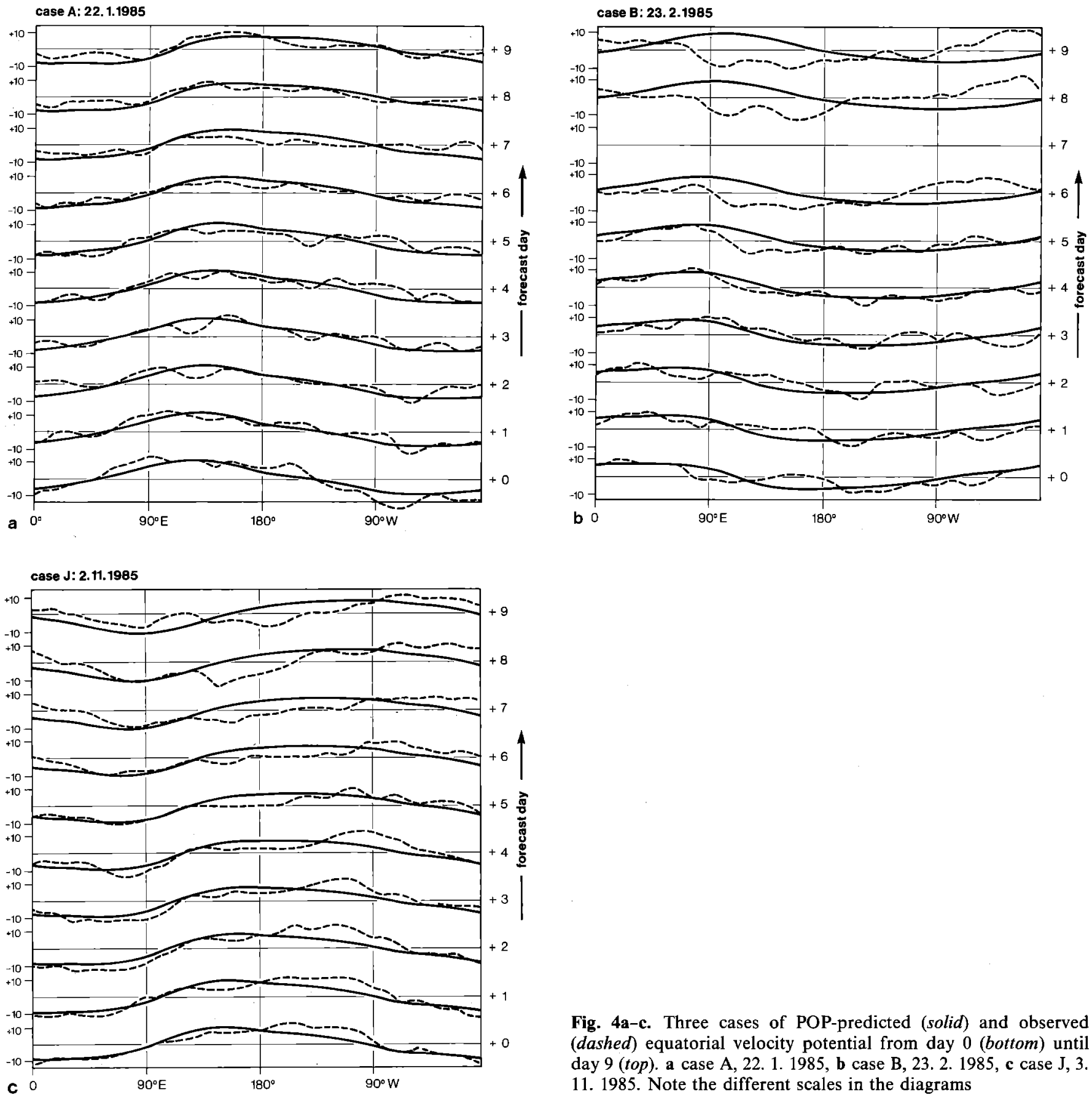

Fig. 4a-c. Three cases of POP-predicted (solid) and observed (dashed) equatorial velocity potential from day 0 (bottom) until day 9 (top). a case A, 22. 1. 1985, b case B, 23.2. 1985, c case J, 3. 11. 1985 . Note the different scales in the diagrams

rial $\chi$-field. Other cases are discussed in the third section when considering the skill of the two GCMs. The choice of 1985 is random; the 1985 data did not enter the fit of the forecast scheme.

The anomaly correlation skill-scores $\mathscr{A}$ for the ten cases are given in Table 1 . Generally the $\chi$-field POP forecasts have large $\mathscr{A}$ values if the POP index POP forecast was classified as "good" in SX (1st row in Table 1). The "false" POP index forecasts, $\mathrm{C}$ and $\mathrm{H}$, often have negative anomaly correlation coefficients. The cases $A, B, G$ and $J$ are discussed in some detail.

In case $G$, a mostly negative anomaly correlation skill score goes along with a "good" POP index POP forecast. This result is due to the fact that, at that time,

the 30- to 60-day oscillation accounted for only a small part of the spatial variance of the $\chi$-field, as can be deduced from the very small $\mathscr{A}(0)=0.04$ at the initial time.

For three of the ten forecasts, A (22.1.1985), B (23.2.1985) and $J(2.11 .1985)$, the POP-predicted and the observed values of $\chi$ are plotted for day 0 and the subsequent 9 days (Fig. 4). The POP-predicted $\chi$-fields are linear combinations of $\mathfrak{p}_{1}$ and $\mathfrak{p}_{2}$; as such, they have to exhibit a smooth wave number 1 pattern, and the actually observed small-scale features cannot be predicted by the POP forecast scheme.

In case A, the POP index is large and its forecast was classified as "good" throughout the first 10 days. 
The observed evolution is fairly uniform; the maximum and the minimum of the velocity potential field propagate slowly eastward. The location of $\chi=0$ in the eastern hemisphere migrates somewhat slower than the zero in the western hemisphere. This evolution of the large scale pattern is very well predicted by the POP scheme for the entire forecast time (Fig. 4a).

The second case, $\mathrm{B}$, is somewhat unexpected: according to the POP index, the 30- to 60-day oscillation exhibited a regular eastward propagation in the first few days. But after 5 days the oscillation changed direction and propagated westward. This finding is substantiated by a visual inspection of the $\chi$-fields (Fig. 4b). Not unexpectedly, the POP index POP forecast was correct in the first phase, but wrong in the second phase. A similar result is obtained for the $\chi$-field POP forecast (Table 1 and Fig. 4b): the predicted fields continue also after day 5 to travel smoothly eastward. We will return to this case in the third section.

In the third example, case $\mathrm{J}$, the $\chi$-field POP forecast is less successful than in case A. Initially, the observed and predicted large-scale patterns are quite similar but after a few days the phase of the predicted 30- to 60day oscillation significantly lags behind the phase of the observed oscillation (Fig. 4c) - a fact that was already visible in the POP index POP forecast (Fig. 9j of SX).

From the examples, it is concluded that, in general, a skillful POP forecast of the POP index is associated with a skillful POP forecast of the broad patterns of the equatorial velocity potential field. If, however, the equatorial $\chi$-field is not dominated by a zonal wave number 1 spatial scale and if it is not temporally coherent (that is, if the 30- to 60-day oscillation is not dominant) the POP forecast fails.

The correlation skill score derived from the entire ensemble

The entire ensemble of $\chi$-field POP forecasts consists of daily forecasts prepared for the period from May 1984 through April 1989. The operational analyses prepared by NMC serve as initial and verifying data. Even though a major change in the NMC analysis procedure was introduced in May 1985 (Trenberth and Olsen 1988), no inhomogeneities in the data set were apparent. Less than $10 \%$ of days were disregarded because the analysis of $200 \mathrm{mb}$ velocity potential was not available. Note that the original POP model was derived from data from May 1986 through April 1988 only. Therefore $60 \%$ of the data used to compute the skill scores represents independent data. The forecasts obtained for the training sample, May 1986 through April 1988 , were not systematically better than for the independent data.

The skills of the POP forecast and of the persistence forecast of the equatorial velocity potential fields are quantified by the correlation skill score $\mathscr{S}$ derived from the entire ensemble (Fig. 5). The POP forecast is inferior to persistence as a "nowcaster" but from day 3

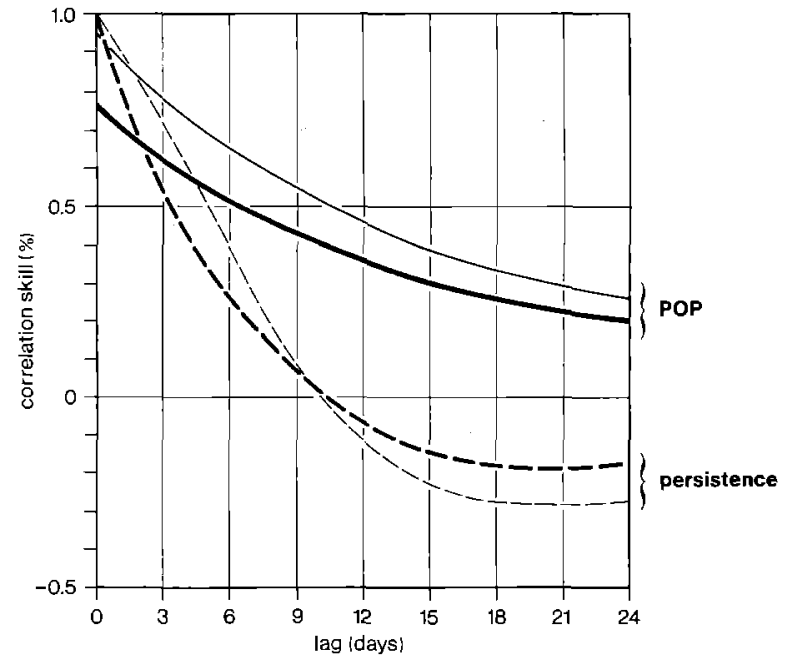

Fig. 5. Correlation skill scores $\mathscr{S}$ of forecasts of equatorial velocity potential (heavy lines) and of the POP index (light lines). A forecast with $\mathscr{S} \leq 0.5$ is considered useless whereas $\mathscr{S}=1$ indicates a perfect forecast. Solid lines: Forecasts prepared by the POP method. Dashed lines: Forecasts made by persistence

onward the POP scheme is a better forecaster. The 0.5 level of $\mathscr{S}$ is reached after 7 days and persistence loses skill after 3 days. The negative scores in the 15-25 day range for persistence is an other indicator of the dominance of the 30- to 60-day oscillation.

The skill obtained for the $\chi$-field POP-forecast (heavy lines in Fig. 5) is less than those of the POP index POP forecast (thin lines). For as long as the persistence forecast is not completely useless, a similar decrease of skill is found for persistence. This deterioration, which accounts for a loss of skillful prediction time of the order of 3 days (POPs) and 2 days (persistence), is not unexpected. The index describes only the 30- to 60-day oscillation (with only two degrees of freedom) whereas the variability of the equatorial velocity potential field is due not only to the 30- to 60-day oscillation but also to high-frequency and small scale features.

It is interesting to see if the skill depends on the strength of the signal. To examine this aspect, the spatial variance, $\sigma_{\chi}^{2}$, of the $\chi$-field, is introduced as an indicator of the strength of the signal. With this measure two subsets are defined:

$$
\begin{array}{ll}
\text { "strong" signals } & \sigma_{\chi}^{2} \geq\left\langle\sigma_{\chi}^{2}\right\rangle \\
\text { "very strong" signals } & \sigma_{\chi}^{2} \geq \frac{3}{2}\left\langle\sigma_{\chi}^{2}\right\rangle
\end{array}
$$

With $\left\langle\sigma_{x}^{2}\right\rangle$ the ensemble average of spatial variance is abbreviated. The correlation skill scores $\mathscr{S}$ obtained for these two subsets and for the entire ensemble are shown in Fig. 6a. Quite clearly a stronger signal is more predictable than a weaker signal. In the "strong" ("very strong") subset $\mathscr{S}$ passes the 50\% level after 11 (20) days. Similarly to the complete ensemble, the subsets exhibit a larger skill for persistence than for the forecast on day zero and day 1 , but from day 2 onwards the POP technique scores better (not shown). 


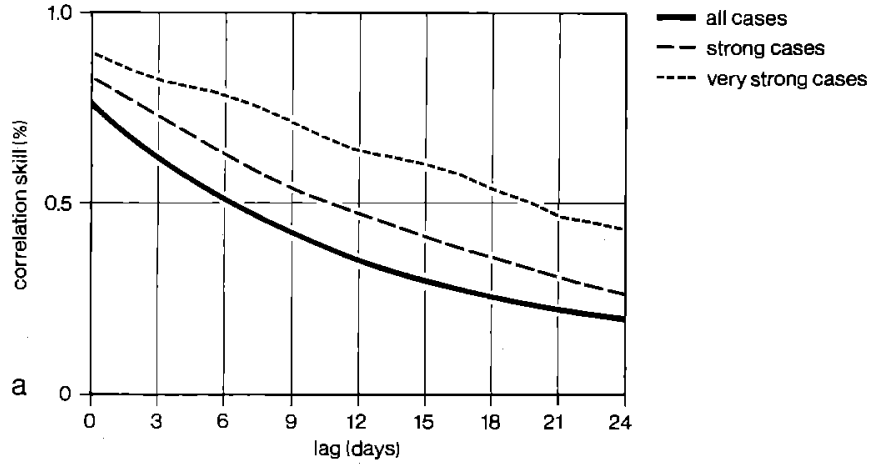

Fig. 6a, b. Correlation skill scores $\mathscr{S}$ of POP forecasts of equatorial velocity potential. A forecast with $\mathscr{S} \leq 0.5$ is considered useless whereas $\mathscr{S}=1$ indicates a perfect forecast. The numbers are derived from approximately 1600 forecasts from May 1984

In Fig. 6b, the correlation skill score $\mathscr{S}$, stratified by season, is shown. In this case, too, the POP forecast scores better than the persistence after 3 days (not shown). $\mathscr{S}$ shows a marked annual cycle: the largest scores are obtained in the northern winter $(\mathscr{S}=0.50$ at day 12) and spring $(\mathscr{S}=0.50$ at day 8$)$; least skill is in the northern summer $(\mathscr{S}=0.50$ at day 4$)$ and fall $(\mathscr{S}=0.50$ at day 7$)$.

\section{POP forecast of area averaged $O L R$}

It was shown in SX that there is a close relationship between the POP index, derived from equatorial velocity potential, and equatorial OLR, in particular in the eastern hemisphere. Therefore, this parameter is potentially predictable by the POP forecast method.

For three areas, one at the equator $\left(75^{\circ}-95^{\circ} \mathrm{E}, 5^{\circ} \mathrm{N}-\right.$ $\left.5^{\circ} \mathrm{S}\right)$, one near Darwin $\left(125^{\circ}-149^{\circ} \mathrm{E}, 10^{\circ}-20^{\circ} \mathrm{S}\right)$ in the Southern Hemisphere, and one in the Northern Hemis-

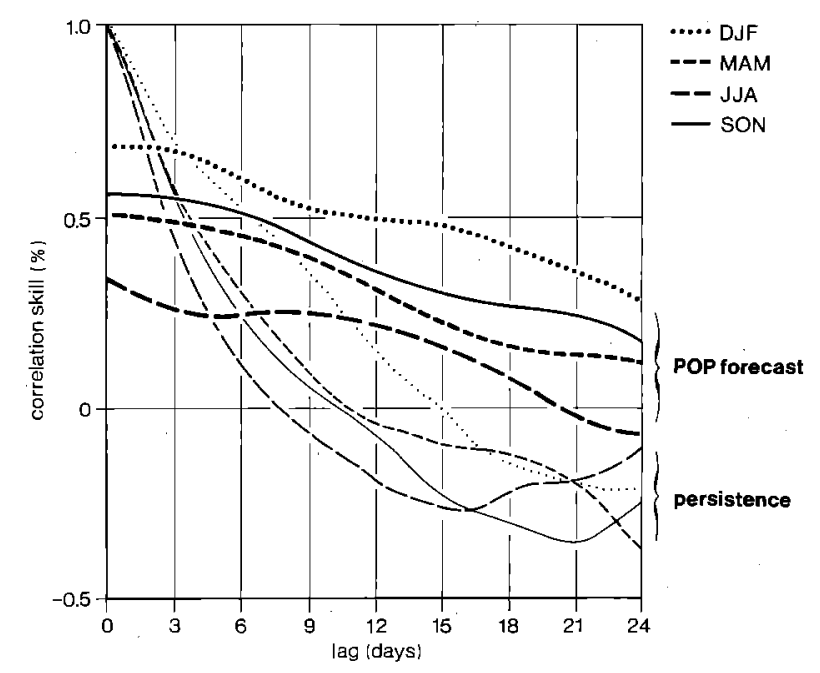

Fig. 7. Correlation skill score $\mathscr{S}$ of the POP and the persistence forecast of outgoing long wave radiation (OLR) averaged over $75^{\circ}-95^{\circ} \mathrm{E}, 5^{\circ} \mathrm{N}-5^{\circ} \mathrm{S}$. The skill score is stratified according to season: December/January/February (DJF), MAM, JJA and SON

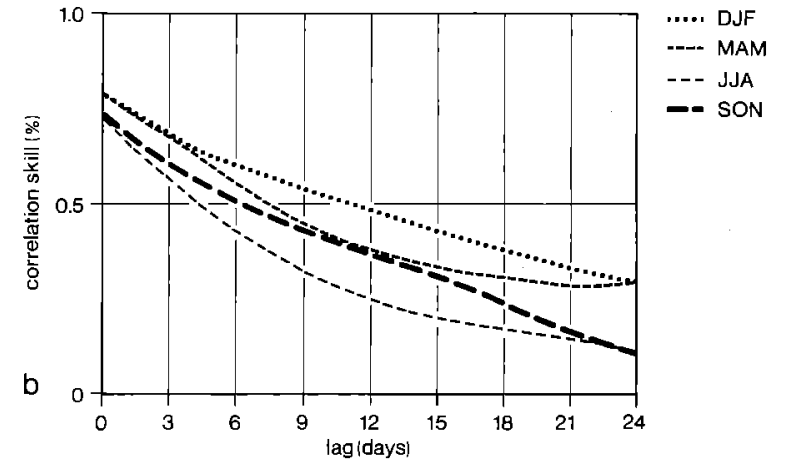

through April 1989. a All data, and the subsets of strong and very strong signals. b Stratification by seasons: DJF (December/January/February), MAM, JJA and SON

phere $\left(115^{\circ}-130^{\circ} \mathrm{E}, 10^{\circ}-20^{\circ} \mathrm{N}\right)$, associated correlation patterns, $\mathfrak{x}_{1}$ and $\mathfrak{x}_{2}$, were derived. The three areas were chosen because of their maximum contribution to the variance in the 30- to 60-day band (Weickmann et al. 1985; Knutson et al. 1986).

The correlation skill scores $\mathscr{S}$ obtained for the two off-equatorial areas are low, in all seasons, for the POP forecast and for the persistence forecast even though the POP forecast exhibits a larger skill than persistence after a few days (not shown). At the equatorial location, however, the POP forecast performs fairly successfully, in particular in DJF; the $\mathscr{S}=0.50$ level is passed after 12 days, which is considerably better than the 7 days obtained by persistence (Fig. 7).

The failure of the off-equatorial OLR POP forecast might be due to the fact that the basic POP model, derived by SX, was established as an all-year model disregarding the large systematic seasonal differences introduced by the Indian monsoon and the Australian monsoon.

\section{GCM forecasts of the equatorial velocity potential}

Experimental extended range forecasts were performed with two GCMs. All cases are northern winter cases. These experiments were mainly made with the purpose of studying problems of extratropical predictability. However, it is worthwhile examining the models' capability of predicting the state of the 30- to 60-day oscillation, in terms of its POP index, and in terms of the anomalous equatorial velocity potential field. The anomalies are defined as the forecast data minus the annual cycle minus the model's systematic errors. The POP indices of the GCM predictions are derived by projecting the forecast $\chi$-anomalies on the adjoint POPs.

In the next section, the NCAR T31 CCM forecasts are considered in some detail, and in the following section, the performance of the CNRM T42 GCM is briefly described. 


\section{The NCAR T31 CCM forecasts}

With the NCAR T31 CCM, a systematic experiment on the model's performance was made (Baumhefner 1989). For nearly every December 1, January 1 and January 30 from 1978 through 1989, a Monte Carlo set of forecasts was conducted. All forecasts were initialized with the standard operational NMC analysis. In this paper, only the control forecast of each set is used.

The model exhibits an initial drift reflecting the inconsistencies of the initial state and the model's dynamics. This trend is removed, together with the annual cycle, by forming averages for the forecast days 0 to +31 from all integrations which are initialized on the same day in the year.

First, two individual forecasts are discussed in some detail, and after that the mean anomaly correlation skill score $\overline{\mathscr{A}}$ and the ensemble correlation skill score $\mathscr{S}$ are derived.

\section{Case studies}

The $\chi$-field NCAR CCM prediction from 30 January 1985 is a very successful forecast (Fig. 8a), with anomaly coefficients $\mathscr{A}>50 \%$ throughout the first 9 days (Table 2). The observed and predicted spatial patterns are similar in the first few days; some differences appear after about 6 days. The GCM forecast seems to be slightly more variable than the observed field. Interestingly, the $\chi$-field POP forecast (Fig. 8b) is also very good, and scores $\mathscr{A} \geq 75 \%$ during the first 9 days (Table 2).

The forecast of the complex POP index $\mathbf{z}$ may be given in terms of a dial diagram. If the complex index is written in polar coordinates, $\mathbf{z}=r \cdot \exp (i \phi)$, the amplitude $r$ represents the present strength of the oscillation. The phase $\phi$ is connected with a longitudinal position of the minimum of the propagating spatial pattern. In Fig. 9 the phases associated with Darwin and with the Dateline are marked. A more detailed diagram is given in SX.

The trajectory in the two-dimensional index-plane, as predicted by the GCM and the POP scheme and as observed, is shown as a dial diagram in Fig. 9 for the 30 day forecast from 30 January 1985 . Throughout all February 1985 , the 30 - to 60 -day oscillation was strong; its phase, in terms of the spatial minimum, travelled regularly from Africa to about the Dateline within 20 days. After 26 February, however, the oscillation changed its direction and moved westward for a few days (see also Fig. 1a, and Fig. 9b in SX). The GCM was very successful in capturing the development in the first 25 days (the deterioration after 6 days was intermittent only) including the initial intensification. The model failed, however, to forecast the westward progression in late February. Similarly to the dynamic GCM forecast, the statistical POP scheme performed well within the first 25 days but failed at the end of the month (see also in the second section).

Another example is shown in Fig. 10: the GCM and the POP forecasts from 1 December 1988. The 30- to 60-day oscillation is weak in the initial state and later also; there was no well organized zonal wave number 1 pattern in the observed state. It is, therefore, not surprising that this forecast is worse than the previous one. The minimum $\mathscr{A}$ of the GCM forecast is only $11 \%$ (day 8), and the POP forecast even scores negative numbers $(\mathscr{A}=50 \%$ on day 8$)$. On day 1 the GCM has generated rather strong small-scale features which fade

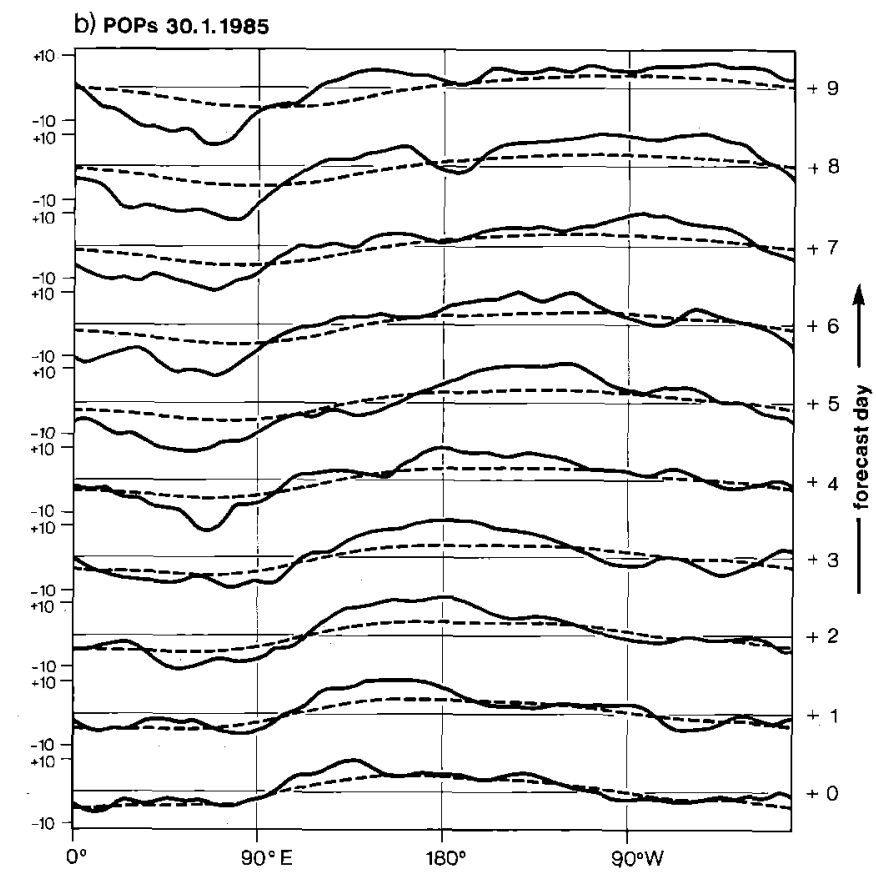

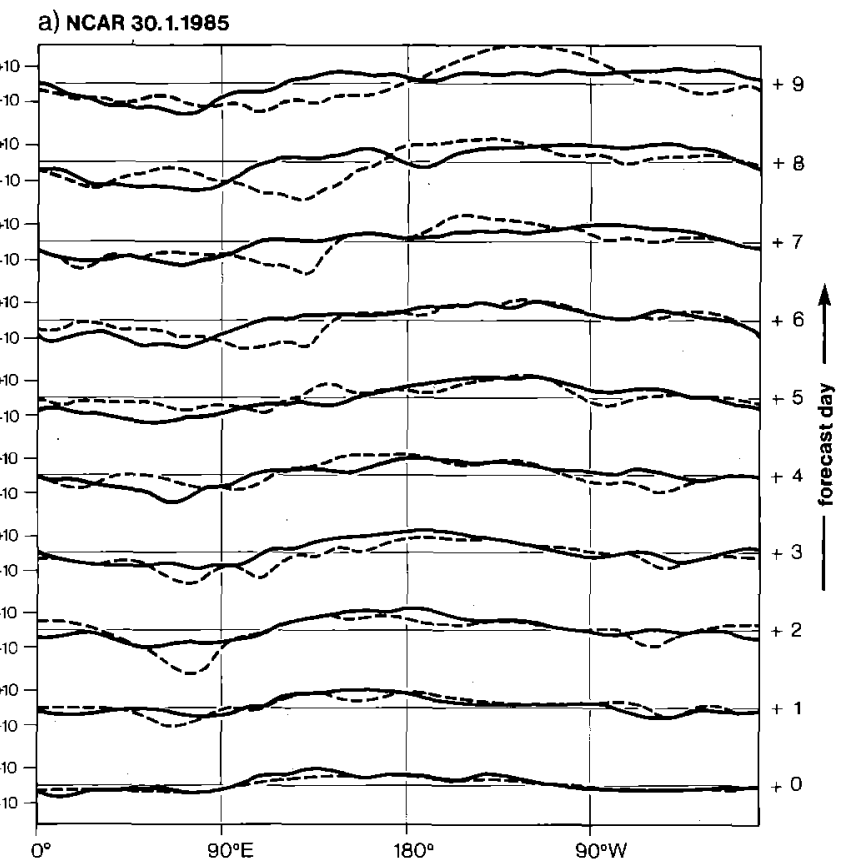

Fig. 8a, b. Predicted (dashed) and observed (solid) evolution of equatorial velocity potential field from day 0 (bottom) until day 9 (top). Day 0 is 30 January 1985. a NCAR T31 CCM forecast, b POP forecast. Note the different scales in the two diagrams 
Table 2. Anomaly correlation coefficients $\mathcal{A}$ of $\chi$-field forecasts prepared by the NCAR CCM and by the POP method. The entry $* *$ marks missing observations. If there is an initial interval with $\mathscr{A} \geq 50 \%$ the numbers are given in italics, indicating a skillful forecast. The cases are ordered after the initial $\mathscr{A}(t, 0)$ of the POP forecast

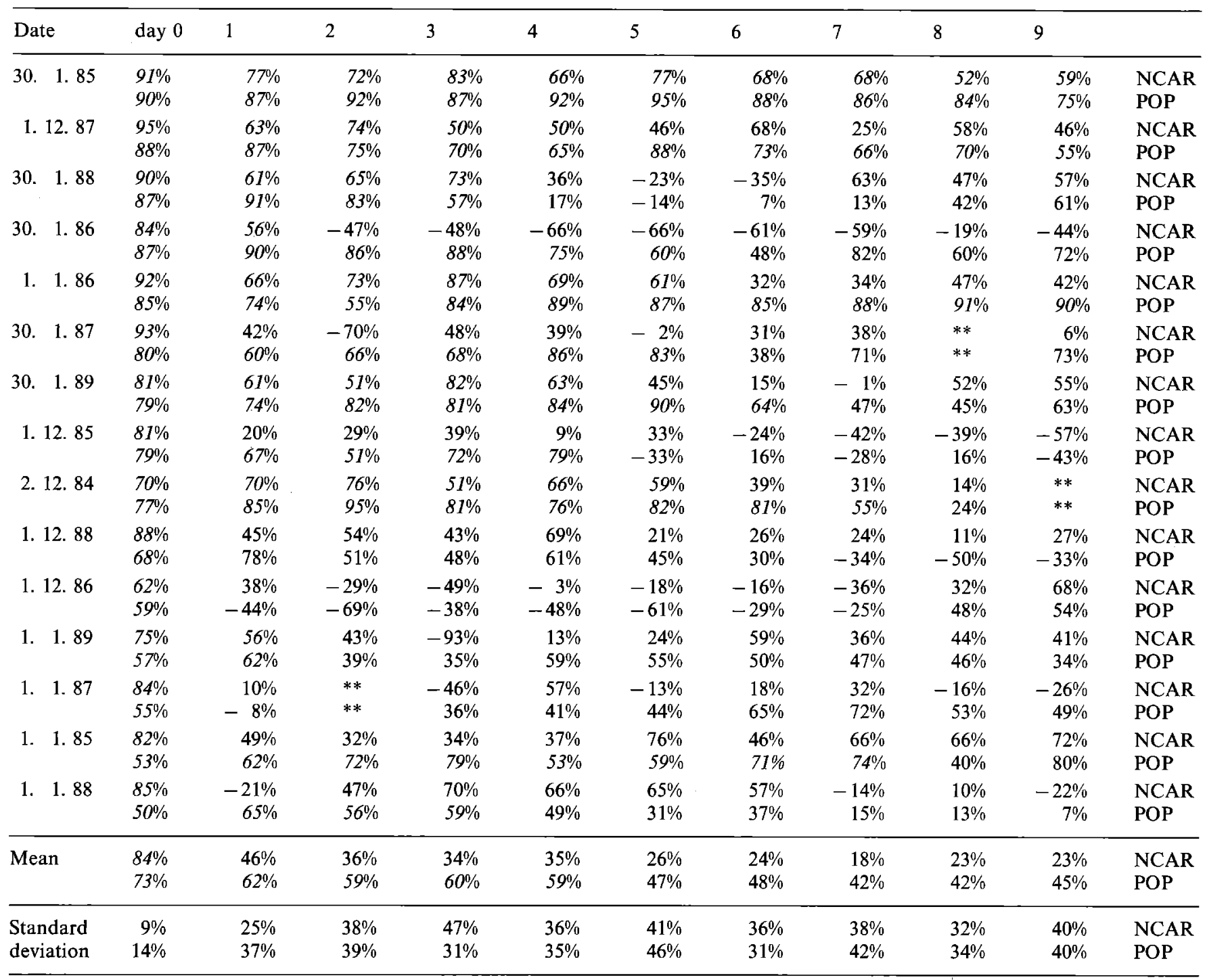

away in the following days. In the dial diagram of the POP index (Fig. 11), it is evident that the GCM predicted 30- to 60-day oscillation propagates too quickly, whereas the POP index POP forecast is fine with respect to the amplitude but the predicted phase considerably lags the real phase. Consistent with the weakness of the 30- to 60-day oscillation, the POP forecast of the $\chi$-field is without skill after a few days. Thus, the basic assumption of the POP forecast, the dominance of the 30- to 60-day oscillation, is violated.

\section{Ensemble skill scores}

The mean anomaly correlation coefficients $\overline{\mathscr{A}}$ of the equatorial velocity potential fields, calculated for the days 0 to +9 of all NCAR CCM forecast experiments and, in parallel, of the POP forecasts, performed for the same dates, are given at the bottom of Table 2. The
If $50 \%$ level is passed by the GCM on day 1 but by the POP scheme after day 4.

In terms of the ensemble correlation skill score $\mathscr{S}$, the numerical model is better than the empirical model only on day 0 (Fig. 12). The correlation skill score $\mathscr{S}$ of the POP is larger than $50 \%$ through day 9 , whereas the GCM is $48 \%$ already on day 1 . The noisy character of the $\mathscr{S}$-curves indicates that an ensemble of only 15 forecasts is not sufficient to yield a reliable estimate of the true skill scores.

\section{The T42 CNRM model}

With the T42L20 version of the CNRM model (Coiffier et al. 1987; Geleyn et al. 1988), experimental lagged average forecasts were performed. Initialized ECMWF analyses interpolated on the model grid were used as initial conditions. For each of 9 different northern 


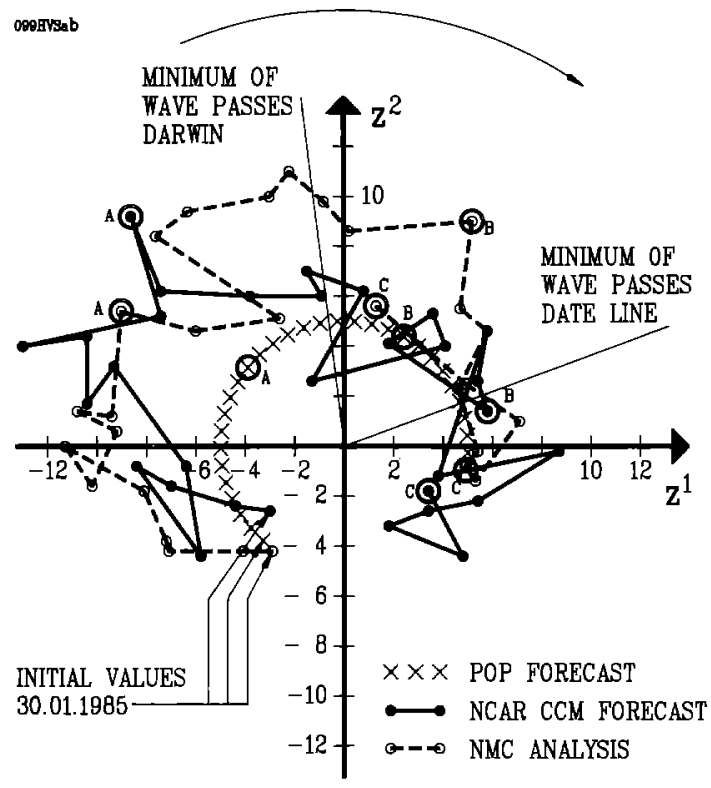
A: 10.02 .1985
B: 20.02 .1985
C: 01.03 .1985

Fig. 9. Dial diagram of the analysis and 30-day predictions of the bivariate POP index in the index-plane. Day 0 of the NCAR T31 CCM and POP forecasts is January 30, 1985. The POP forecast scheme implies a clockwise rotation of the trajectory. The indicated geographic positions mark the location of the minimum of the spatial pattern, if the $\left(z_{1}, z_{2}\right)$ spans the respective angle with the horizontal axis (see also Figs. 2 and 9 in SX). The days 11, 21 and 30 are marked as $A, B$ and $C$. Note that a few NMC analyses are missing winter dates, a set of 5 forecasts were integrated from initial conditions which were 12 hours apart from each other. So, the day 0 "nowcast" is actually the mean of the analysis of day 0 , and of four forecasts prepared with initial conditions $12,24,36$ and 48 hours earlier. The observed annual cycle has been removed from the raw data and also the model's systematic error, by subtracting the 15 day running mean error. This estimate of the model's systematic error is certainly not very accurate because of the small sample size. Due to logistic problems, day $0 \chi$-fields simulated by the model are not available.

The averaged anomaly correlation coefficient $\bar{Z}$ as a function of lag is given in Table 3 and the ensemble correlation skill $\mathscr{S}$ is shown in Fig. 13. Because of the small sample size, both measures of skill, $\mathscr{\mathscr { A }}$ and $\mathscr{S}$, are not strictly monotonic functions of the lag $\tau$ but quite variable. Nevertheless, the statistical approach appears much better than the dynamical model. This finding is substantiated by inspection of the individual cases (not shown). The CNRM GCM scores better than the POPs only if there is no well-defined wave number 1 pattern present in the initial state.

The characteristic eastward propagation of the largescale signal often does not occur in the GCM data. An example is given in Fig. 14, which shows the observed and predicted temporal evolution of the POP index from 16 November 1987 onwards. Initially the POP index is strong, and it travels clockwise, indicating regular eastward propagation, in the two-dimensional plane. This behavior is correctly predicted by the POP model. The CNRM model is capable of predicting the first few days, but the signal is then quickly dampened.
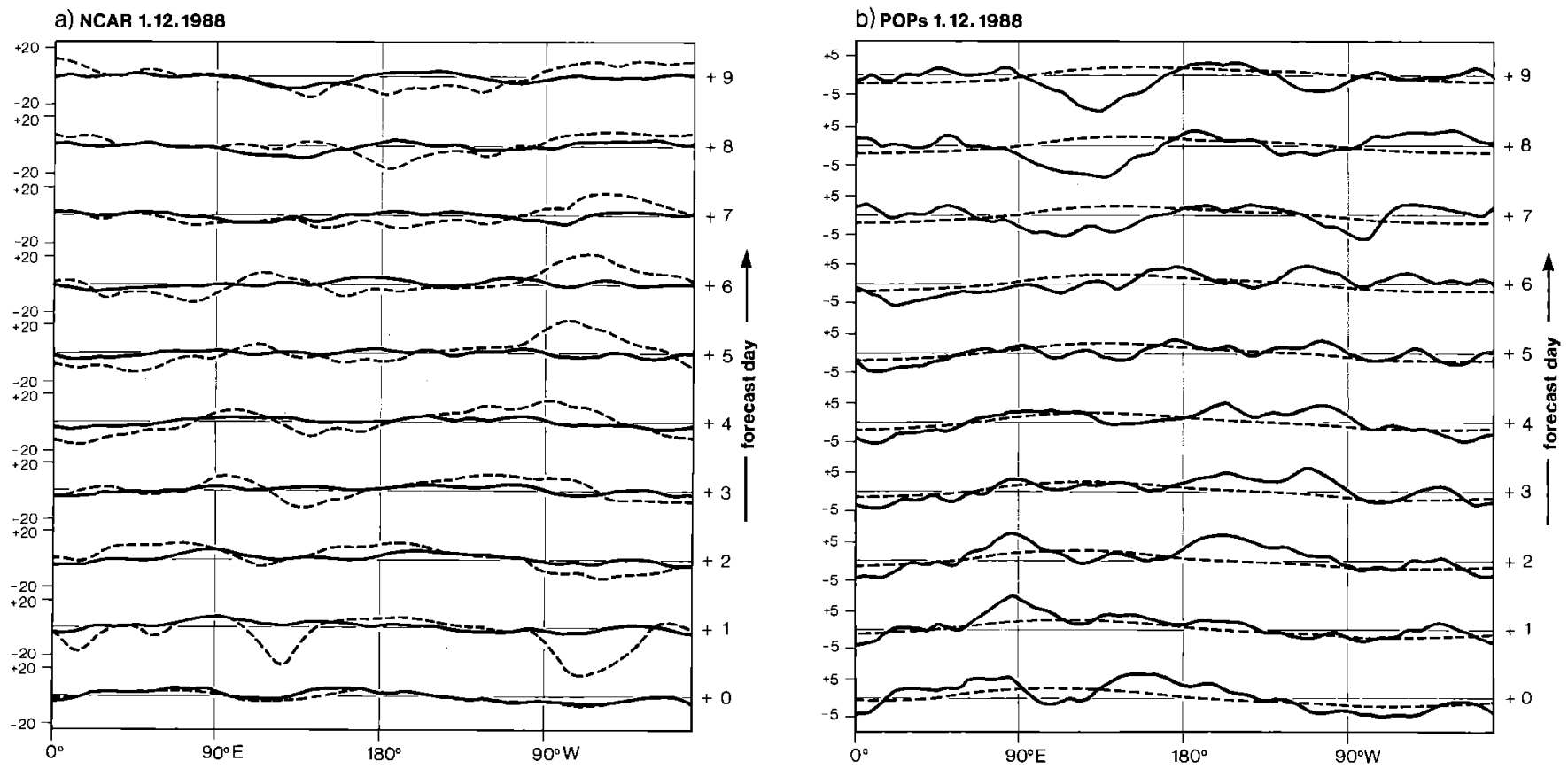

Fig. 10a, b. Predicted (dashed) and observed (solid) evolution of equatorial velocity potential field from day 0 (bottom) until day 9 (top). Day 0 is 1 December 1988. a NCAR T31 CCM forecast, b POP forecast. Note the different scales in the two diagrams 


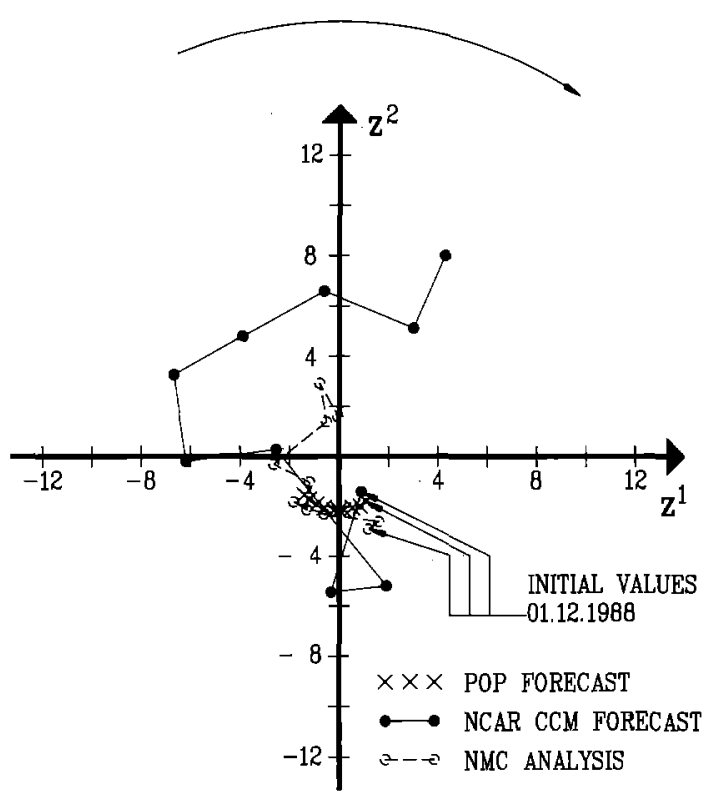

Fig. 11. Analysis and 9 day predictions of the bivariate POP index in the index-plane. Day 0 of the NCAR T31 CCM and POP forecasts is December 1,1988

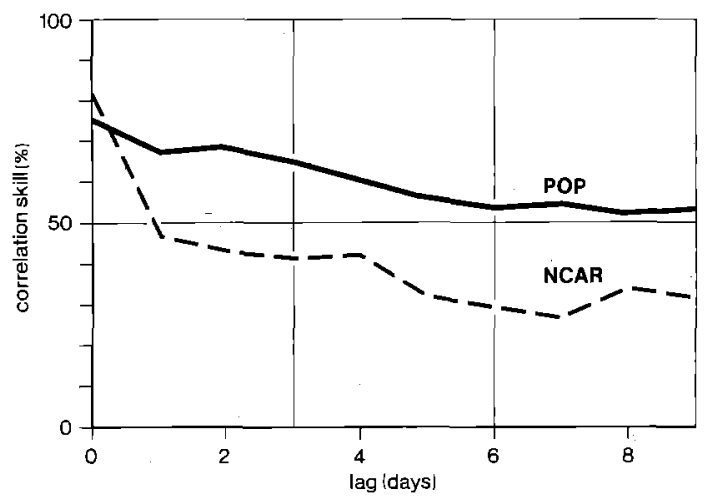

Fig. 12. Ensemble correlation skill $\mathscr{P}$ derived from 15 forecasts prepared with a the NCAR T31 CCM and the POP method. A forecast with $\mathscr{S} \leq 0.5$ is considered useless whereas $\mathscr{S}=1$ indicates a perfect forecast. Note that the curve for the POP forecast deviates from that in Fig. 5; only the 15 cases covered by the NCAR T31 CCM experiment have been used to estimate the skill

\section{Summary and discussion}

In Part I (Storch and Xu 1990) it was found that the POP scheme is remarkably skillful in predicting the index of the 30- to 60-day oscillation. In this Part II we considered equatorial velocity potential $\chi$ at $200 \mathrm{mb}$ and questioned whether this parameter, and OLR, might be predicted skillfully successfully by the POP method. The POP technique was indeed successful in forecasting the $\chi$-field along the equator and OLR in the equatorial Indian Ocean area.

It was found that the POP forecast is most successful in DJF and when the initial 30- to 60-day oscillation signal is strong. Possibly, these two facts are not independent of each other; the annual cycle of the skill (Fig. 6b) might reflect the annual cycle of the mean strength of the signal shown in Fig. 15. This is at least partly true: the mean signal is strongest in the northern winter season when the skill is largest.

There is one more potential candidate that might be responsible for the annual cycle of the scheme's skill: the basic statistical model was fitted with all-year data disregarding the strong monsoon signals. Recently, a generalization of the POP method, named cyclostationary POP analysis, has been developed (Blumenthal 1991 (submitted); Alonso et al. 1990, personal communication). It might be rewarding to redo our POP analysis with this improved technique, which explicitly considers seasonality.

The POP scheme is considerably better than the persistence forecast. If there is a significant 30- to 60-day oscillation in the initial state the $\chi$-field POP forecast also scores better than dynamical forecasts made with the NCAR T31 CCM and with the CNRM T42 GCM. In some cases, the NCAR CCM was also quite successful.

The fact that the GCMs are not overall superior to the simple "persistence of motion" POP forecast is in some sense encouraging. It should be possible to improve the dynamical models, or their initializations, so that they are at least as good as the POPs. For both models, no particular attention was paid to the initialization of the tropical circulation. The fact that, the lower resolution NCAR T31 model yields better results than the higher resolution CNRM T42 model, indicates that a model's physics might be more important in reproducing realistically large-scale tropical variations than the model's horizontal resolution.

It is interesting to examine the GCM's forecast skill as a function of the initial strength of the 30- to 60-day oscillation. This initial strength is measured by the correlation $\mathscr{L}_{\text {POP }}(t, 0)$ between the $\chi$-field POP "nowcast", $\hat{\mathfrak{x}}(t+0)$, and the verifying analysis, $\mathfrak{x}(t)$, i.e., the initial anomaly correlation coefficient $\mathscr{A}$.

Table 3. Mean anomaly correlation skill $\mathscr{\mathcal { A }}$ of $9 \chi$-field forecasts prepared by the CNRM T42 GCM and by the POP method. A forecast with $\not{\mathscr{P}} \leq 50 \%$ is considered useless, and $\mathscr{\mathscr { A }}=100 \%$ indicates a perfect forecast

\begin{tabular}{|c|c|c|c|c|c|c|c|c|c|c|c|}
\hline $\begin{array}{l}\text { Prediction } \\
\text { method }\end{array}$ & \multicolumn{11}{|c|}{ Forecast day } \\
\hline POP & $54 \%$ & $45 \%$ & $52 \%$ & $52 \%$ & $50 \%$ & $68 \%$ & $61 \%$ & $60 \%$ & $46 \%$ & $43 \%$ & POP \\
\hline
\end{tabular}




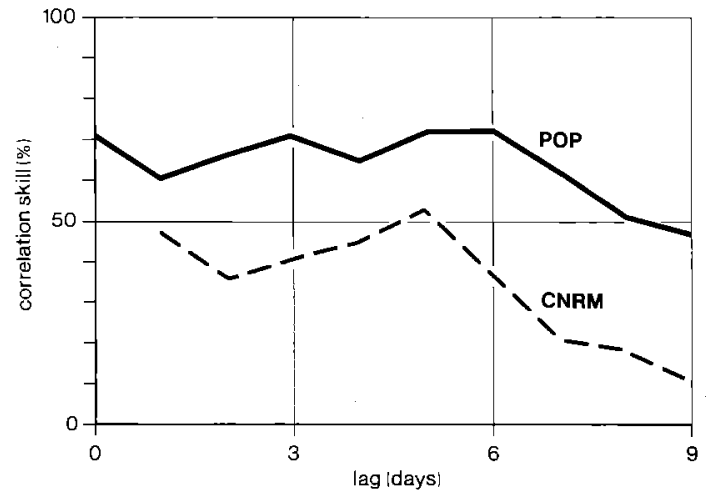

Fig. 13. Ensemble correlation skill $\mathscr{S}$ derived from 9 forecasts prepared with a the CNRM T42 GCM and the POP method. Note that the curve for the POP forecast deviates from those in Figs. 5 and 12 ; only the 9 cases covered by the CNRM T42 GCM experiment have been used to estimate the skill. Owing to the small sample the estimated skill scores are quite variable

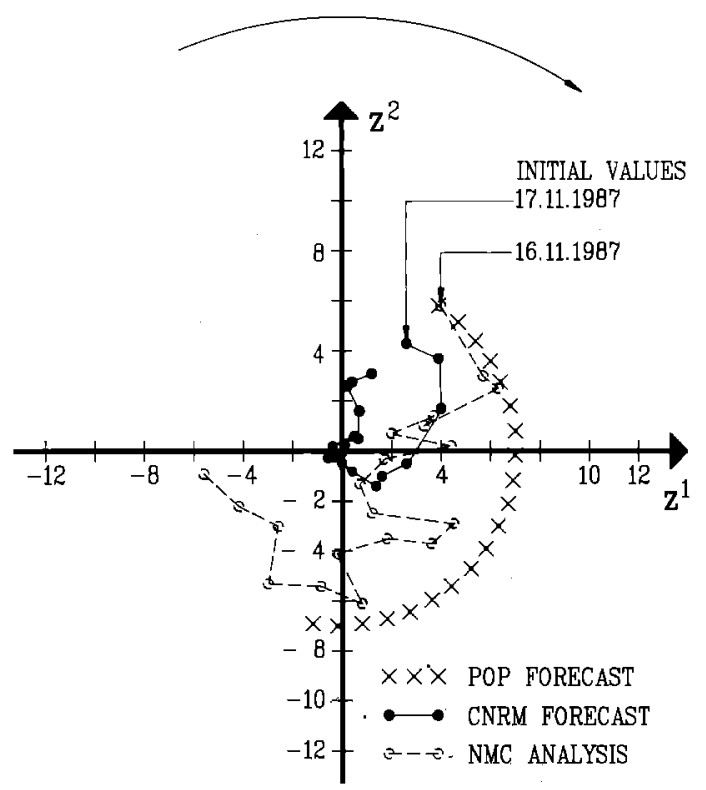

Fig. 14. Analysis (dashed line) and 20 day prediction of the bivariate POP index in the two-dimensional index. Day 0 of the CNRM T42 GCM (solid line) and POP (stars) forecasts is 11 November 1987. Note that day 0 is not included in the GCM forecast so that the first point on the GCM curve is 17 November

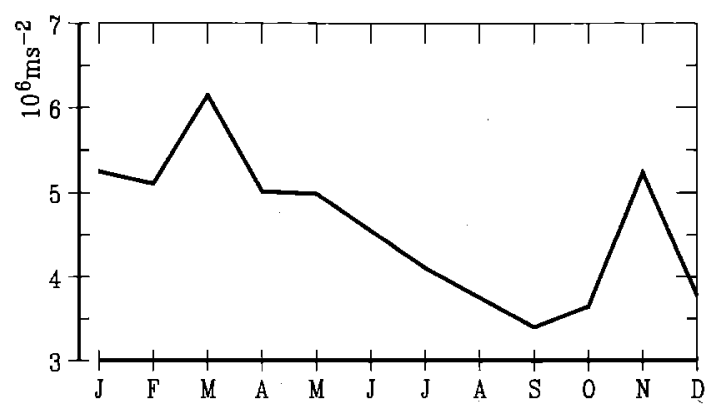

Fig. 15. Annual cycle of the mean strength, $\langle\|z\|\rangle=\langle r\rangle$, of the index of the 30- to 60-day oscillation

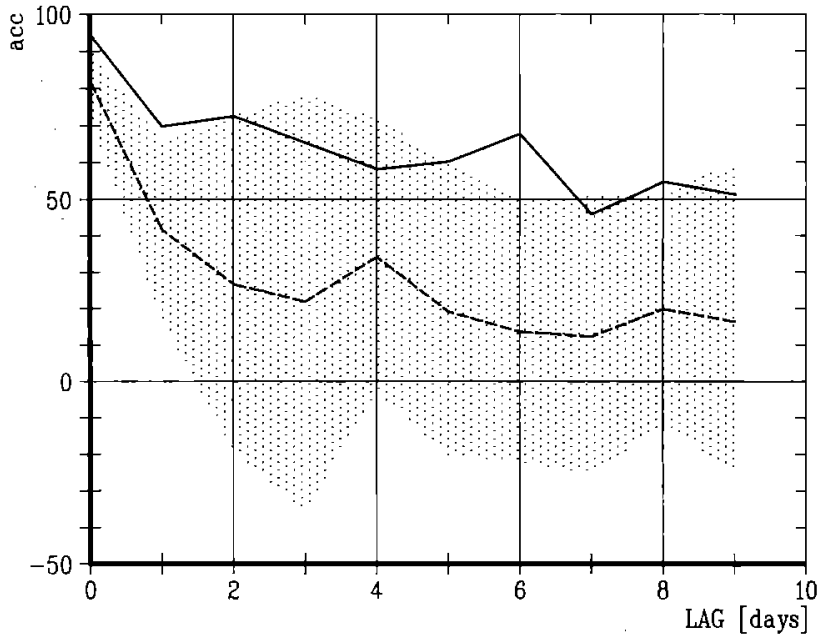

Fig. 16. To demonstrate the tentative finding that the size of the initial $\mathscr{A} / \mathrm{POP}(t, 0)$ is an indicator of the NCAR CCM's skill in predicting the equatorial $\chi$-field, the set of all NCAR CCM forecasts is split up into two subsets: $\Omega_{1}=\left\{t ; \mathscr{S}_{\mathrm{POP}}(t, 0)>88 \%\right\}$ and $\Omega_{u}=\left\{t ; \mathscr{P}_{\mathrm{POP}}(t, 0) \leq 88 \%\right\}$. The dashed line is the mean anomaly correlation skill score $\overline{\mathscr{A}}(\tau)$ of $\Omega_{1}$ and the solid line that of $\Omega_{u}$. The hatched area indicates \pm one standard deviation of the $\mathscr{A}$ 's in $\Omega_{1}$. The number of samples is, however, too small to make the differences statistically significant: $\left|\Omega_{1}\right|=13$ and $\left|\Omega_{u}\right|=2$

While no useful relationship was found for the CNRM T42 model, the size of $\mathscr{A}_{\mathrm{POP}}(t, 0)$ appeared to be an indicator of the NCAR CCM's skill in predicting the equatorial $\chi$-field (Table 2). To demonstrate this tentative finding, the set of all NCAR CCM forecasts is split up into two subsets: $\Omega_{1}=\left\{t ; \mathscr{A}_{\mathrm{POP}}(t, 0)<\omega\right\}$ and $\Omega_{u}=\left\{t ; \mathscr{A}_{\mathrm{POP}}(t, 0) \leq \omega\right\}$ with some arbitrary number $\omega$. Then the means of both subsets and the standard deviation of $\Omega_{1}$ are derived for each lag $\tau$. The result for $\omega=88 \%$ is plotted in Fig. 16. Clearly the NCAR CCM's skill in predicting the equatorial velocity potential in the subset $\Omega_{u}$ is better than in the subset $\Omega_{1}$. The number of samples is, however, too small to make this finding statistically significant.

Also, it is worthwhile studying the GCM's skill in predicting the extratropical height fields stratified either by the strength of the 30- to 60-day oscillation in the initial field or by the model's capability of correctly forecasting the oscillation. An analysis of the NCAR CCM performance is presently underway. In the case of the CNRM T42 GCM, no useful relationship between extratropical predictability (in terms of the 15- or 30-day mean field) and the tropical flow was found.

\section{Appendix}

\section{POP analysis}

The POP analysis (Hasselmann 1988; Storch et al. 1988; Storch et al. 1990) is based on the statistical assumption that the considered vector time series $\mathfrak{r}$ might be modeled by a first order autoregressive vector process, i.e., that the space-time statistics of the process: 
$\mathfrak{z}(t+1)=\mathfrak{A} \cdot\{(t)+$ noise

are an adequate approximation of the space-time statistics of the time series $\mathfrak{r}$. The matrix $\mathfrak{Z}$ is estimated from the data. Then, the Principal Oscillation Patterns (POPs) are the eigenvectors of $\mathfrak{X}$ which are, in general, complex. Expanding a vector $\mathfrak{x}(t)$ into the eigenvectors $\mathfrak{p}$ yields the representation:

$\mathfrak{x}(t)=\sum_{i} \mathrm{z}^{i}(t) \mathfrak{p}_{i}$

with the POP coefficients $\mathrm{z}^{i}(t)$. Insertion of (B) into (A) yields a time evolution equation for the POP coefficients, with $\lambda_{i}$ denoting the eigenvalue connected with the eigenvector $\mathfrak{p}_{i}$

$\mathbf{z}^{i}(t+1)=\lambda_{i} \cdot \mathbf{z}^{i}(t)+$ noise

if the eigenvalue is complex then the POP coefficients are also complex and equations (C) describes the characteristic evolution mentioned in the Introduction

$\cdots \rightarrow-\mathfrak{p}_{1} \rightarrow \mathfrak{p}_{2} \rightarrow \mathfrak{p}_{1} \rightarrow-\mathfrak{p}_{2} \rightarrow-\mathfrak{p}_{1} \rightarrow \cdots$

\section{POP forecast}

The POP forecast technique is predicting the evolution of the POP coefficients $\mathrm{z}(t)$ by means of $(\mathrm{C})(\mathrm{Xu}$ and Storch 1990; SX). Of course, the unknown noise is set to zero so that the forecast equation is simply:

$\hat{\mathbf{z}}(t+1)=\lambda \cdot \mathbf{z}(t)$

After having identified the eigenvalue, the only problem left is to derive the initial value. In most applications, the data exhibit a substantial day to day (or month to month) variability masking the signal. Thus, it is often necessary to derive the initial value from a weighted average of the observation at day 0 and a few days earlier.

Acknowledgements. The observational data used in this study were supplied by Drs. George Kiladis and Klaus Weickmann at CIRES; the CNRM data were provided by Dr. Michel Deque at CNRM. Tom Mayer and Dennis Joseph of NCAR prepared the data base to process the NCAR CCM results. Helpful comments were given by Jinsong $\mathrm{Xu}$ and Rainer Schnur and one of the anonymous reviewers. The diagrams were thoroughly prepared by Marion Grunert and Doris Lewandowski.

\section{References}

Baumhefner DP (1989) Prediction of forecast skill using Monte Carlo ensembles of 30 day forecasts. Proc. 14th Climate Diagnostics Workshop, Climate Analysis Center, NMC/NWS/ NOAA, WWB Building 606, 5200 Auth Road, Washington DC 20233

Blumenthal MB (1991) Predictability of a coupled ocean-atmosphere model. Submitted to J Climate

Coiffier J, Ernie Y, Geleyn JF, Clochard J, Hoffmann J, Dupont F (1987) The operational hemispheric model at the French meteorological service. J Meteor Sec Japan, special NWP symposium volume: $337-345$

Geleyn JF, Bougeault P, Rochas M, Cariole D, Lafore JP, Royer JF, Andre JC (1988) The evolution of numerical weather prediction and atmospheric modelling at the French weather service. J Theoretical Appl Mech 7:87-110

Hasselmann KH (1988) PIPs and POPs: the reduction of complex dynamical systems using principal interaction and oscillation patterns. J Geophys Res 93:11 015-11021

Knutson TR, Weickmann KM, Kutzbach JE (1986) Global-scale intraseasonal oscillations of outgoing longwave radiation and $250 \mathrm{mb}$ zonal wind during Northern Hemisphere summer. Mon Wea Rev 114:605-623

Madden RA, Julian PR (1972) Description of global scale circulation cells in the tropics with a $40-50$ day period. J Atmos Sci 24:1109-1123

Murphy AH, Epstein ES (1989) Skill scores and correlation coefficients in model verification. Mon Wea Rev 117:572-581

Storch H v, Bruns T, Fischer-Bruns I, Hasselmann KH (1988) Principal oscillation pattern analysis of the 30 - to 60 -day oscillation in a general circulation model equatorial troposphere. $\mathbf{J}$ Geophys Res 93:11022-11036

Storch H v, Weese U, Xu J (1990) Simultaneous analysis of spacetime variability: principal oscillation patterns and principal interaction patterns with applications to the Southern Oscillation. Z Meteor 40:99-103

Storch H v, Xu J (1990) Principal oscillation pattern analysis of the tropical 30- to 60-day oscillation. Part I: definition of an index and its prediction. Clim Dyn 4: 175-190

Trenberth KE, Olsen JG (1988) Evaluation of NMC global analyses: 1979-1987. NCAR Technical Note NCAR/TN$299+$ STR

Weickmann KM, Lussky GR, Kutzbach JE (1985) Intraseasonal (30-60 day) fluctuations of outgoing longwave radiation and $250 \mathrm{mb}$ streamfunction during northern winter. Mon Wea Rev 113:943-961

Tribbia J, Baumhefner DP (1988) Estimates of the predictability of low-frequency variability with a spectral general circulation model. J Atmos Sci 45:2306-2317

Xu J, Storch H v (1990) Predicting the state of the Southern Oscillation using principal oscillation pattern analysis. J Climate 3:1316-1329 